\title{
The Cuban Treefrog (Osteopilus septentrionalis) in Florida ${ }^{1}$
}

\section{Steve A. Johnson ${ }^{2}$}

The Cuban Treefrog, Osteopilus septentrionalis (Figure 1), is native to Cuba, the Isle of Youth (an island province of Cuba also known as Isle of Pines), the Cayman Islands, and the Bahamas. It is an introduced species in Florida, and the earliest confirmed records date to the 1920s in the Florida Keys. The first Cuban Treefrogs in Florida likely arrived as stowaways in shipping crates originating from the Caribbean. By the mid-1970s, they had dispersed throughout most of southern Florida. As of 2007, there appear to be established breeding populations as far north as Cedar Key on Florida's Gulf Coast, Jacksonville on the Atlantic Coast, and Gainesville in north-central Florida (Figure 2). This species continues to expand its range and individuals have been documented in coastal Georgia and South Carolina, as well as the panhandle of Florida in the past few years. Cuban Treefrogs spread by hitchhiking on ornamental plants, motorized vehicles, boats, etc. The Cuban Treefrog is considered an invasive species in Florida.

\section{Identifying Cuban Treefrogs}

The Cuban Treefrog is a member of the frog family Hylidae. All of Florida's treefrogs, including

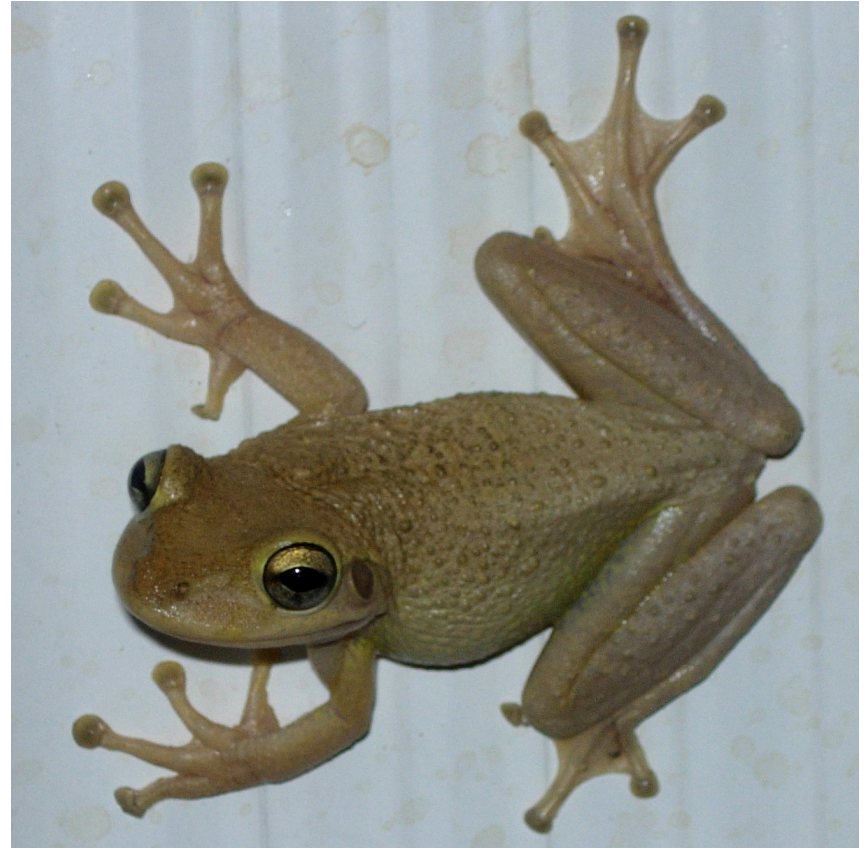

Figure 1. Adult Cuban Treefrog Credits: Steve A. Johnson, University of Florida, 2005

Cuban Treefrogs, have expanded pads on the ends of their toes that allow them to climb trees, shrubs, windows, and buildings. The presence of toe pads can help distinguish treefrogs from other frogs such as toads and aquatic frogs like bullfrogs. Cuban

1. This document is WEC218, one of a series of the Department of Wildlife Ecology and Conservation, Florida Cooperative Extension Service, Institute of Food and Agricultural Sciences, University of Florida. First published: May 2007. Please visit the EDIS Web site at http://edis.ifas.ufl.edu.

2. Steve A. Johnson, Assistant Professor, Department of Wildlife Ecology and Conservation, Gulf Coast Research and Education Center, University of Florida's Plant City Campus, 1200 North Park Road, Plant City, FL 33563. 


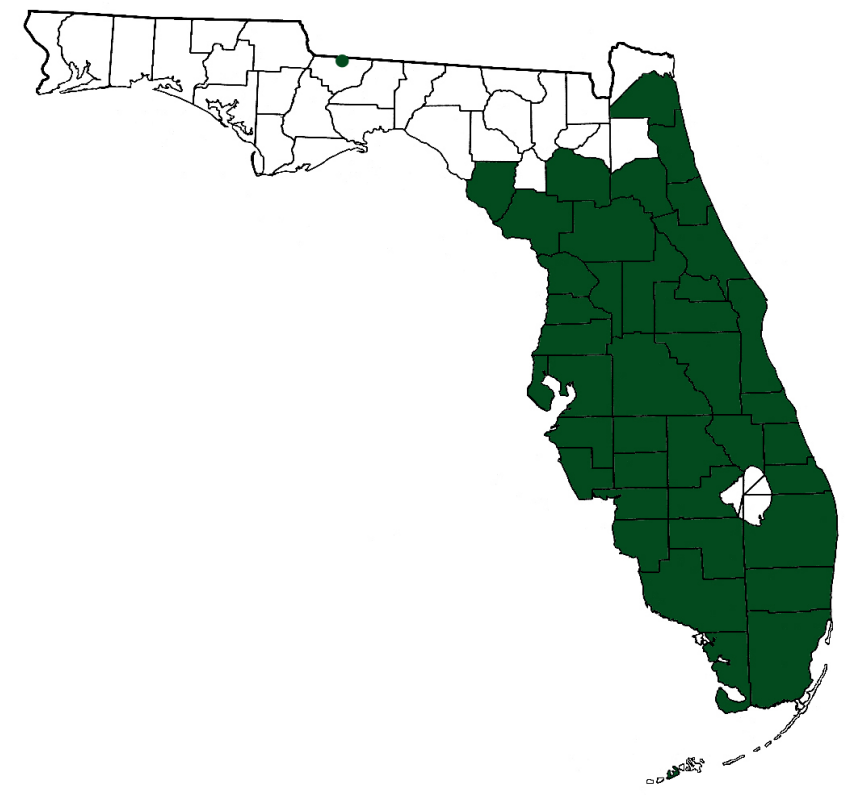

Figure 2. Geographic distribution of Cuban Treefrogs (by county) in Florida Credits: Monica McGarrity, University of Florida, 2007

Treefrogs have exceptionally large toepads as compared to Florida's native treefrogs (Figure 3).

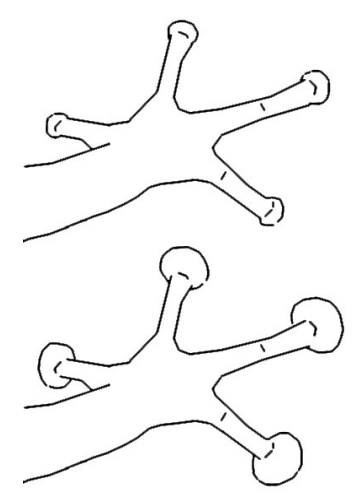

Figure 3. The toepads of the Cuban Treefrog (lower image) are exceptionally large in comparison to the toepads of native treefrog species (upper image). Credits: Monica McGarrity, University of Florida, 2007

Cuban Treefrogs are the largest species of treefrog in Florida, and adult females may exceed 6 inches in length. Most Cuban Treefrogs, however, range from 1-4 inches long. They have very large eyes, giving them a somewhat "bug-eyed" appearance. They have rough or even warty skin, sometimes have a pattern of large wavy markings or blotches on their back, and frequently have stripes or bands on the dorsal surface of their legs. The colors of Cuban Treefrogs vary a lot. Most often they are creamy white to light brown, although they can be green, gray, beige, yellow, dark brown or a combination of these colors. They have a yellowish-colored wash where their front and rear legs are attached to their body.

Several of Florida's native treefrogs superficially resemble Cuban Treefrogs. These frogs, like Cuban Treefrogs, also show considerable variation in colors and markings, but generally are smaller and have smaller eyes. Therefore it can be difficult to distinguish native treefrogs from invasive Cuban Treefrogs. However, there are ways to confidently identify all of Florida's treefrogs and with a little bit of practice anyone can tell them apart. The two native species most likely to be found with Cuban Treefrogs are the Green Treefrog, and the Squirrel Treefrog. Both of these native species are usually colored dull to bright green and have skin that lacks distinct warts. Green Treefrogs grow to about 2.5 inches, have a distinct white line on each side of their body, and have a pointed snout. Squirrel Treefrogs only grow to 1.5 inches, but their colors and markings vary considerably. For details and images of Florida's treefrogs, and to learn how to differentiate them please see the forthcoming University of Florida/IFAS fact sheet on Florida's treefrogs by Dr. Steve Johnson (http://edis.ifas.ufl.edu/TOPIC_A23708620). Also visit the web sites listed at the end of this document to find digital images of Cuban and native treefrogs.

\section{Cuban Treefrog Ecology and "Natural History"}

Cuban Treefrogs are found throughout a variety of natural and human-modified habitats in Florida. Natural habitats invaded by Cuban Treefrogs include pine forests, hardwood hammocks, and swamps. In urban and suburban settings they are most commonly found on and around homes and buildings, and in gardens and landscape plants. They also occur in agricultural settings, orange groves, and plant nurseries. They occur throughout Florida's peninsula (Figure 2), and are continuing to expand their range to the north. The expansion of their range is augmented by the activities of people. Cuban Treefrogs are transported to new areas as stowaways 
on cars, trucks, and boat trailers as well as in ornamental plants and trees that are shipped north from South Florida.

Like our native treefrogs, Cuban Treefrogs are excellent climbers and will climb high into trees where they sleep during the day. They may also be found closer to the ground in small trees and shrubs. They have even been found buried several inches deep in dry soil. Also like our native treefrogs, Cuban Treefrogs are most active at night when they come out from daytime hiding places to feed and reproduce.

Invasive Cuban Treefrogs eat a wide variety of food items, including snails, millipedes, spiders, and a vast array of insects. They are predators of several of Floridas native frogs, and are cannibalistic. They are also known to eat lizards and even small snakes. Fortunately, several species of native snakes will eat Cuban Treefrogs, including rat snakes, Black Racers, Pygmy Rattlesnakes, and Garter Snakes. Owls, crows, and wading birds have also been seen feeding on Cuban Treefrogs.

Cuban Treefrogs breed predominately in the spring and summer. Reproduction is largely stimulated by rainfall, especially warm summer rains such as those associated with tropical weather systems and intense thunderstorms. The number of eggs deposited by a female is related to her size-larger females lay more eggs. A very large female may lay in excess of 15,000 eggs in one season. Cuban Treefrogs are not picky about their breeding sites, as long as the sites lack predatory fish, such as bass and bream. Acceptable breeding sites include isolated wetlands, ditches, decorative ponds, and even swimming pools that are neglected. They also breed in much smaller bodies of water, such as stagnant water in a neglected ice chest, a child's wading pool, or other objects that accumulate water.

Male Cuban Treefrogs have a fairly distinct call that sounds like a squeaking door and has also been described as a "snoring rasp"- -visit the web sites listed at the end of this document to find links to recordings of Cuban Treefrog calls. The vocal sac of the male, which is used to make breeding calls, is located under the chin of the frog and when inflated has the appearance of a "double sac", rather than the single rounded, balloon-shaped sac of our native treefrogs. In addition to their breeding calls, individual males will also call from daytime retreat sites to advertise their presence. This "rain call", as it is sometimes called, can be triggered by light rainfall during the day.

\section{Invasive Cuban Treefrogs and Their Impacts in Florida}

An invasive species is generally defined as a plant, animal or microbe that is found outside of its native range, where it negatively impacts the ecology, economy, or quality of human life. Cuban Treefrogs fit this definition of an invasive species because they were introduced to Florida by the activities of people and they are causing harm to Florida's natural ecosystems and the quality of life of Floridians. They are also causing economic impacts in some places.

\section{Ecological Harm}

There is mounting evidence that Cuban Treefrogs are having negative impacts on Florida's native species and ecosystems. Although they predominately occur around human development, such as urban neighborhoods, Cuban Treefrogs also are able to invade natural areas. In both natural and urbanized settings, Cuban Treefrogs are known to be predators of Florida's native treefrogs (Figure 4). They also are known to eat several additional species of native frogs, lizards, and many types of invertebrates. Many homeowners in Florida report that Cuban Treefrogs appear to have replaced native treefrogs as the dominant frog found around their homes. These same people say that they no longer see native species, such as Squirrel Treefrogs or Green Treefrogs, but only Cuban Treefrogs. Furthermore, lab experiments (conducted by herpetologist Dr. Kevin Smith) have shown that Cuban Treefrog tadpoles are superior competitors with at least two species of native frog tadpoles. Tadpoles of the invasive Cuban Treefrog inhibited the growth and development of tadpoles of our native Southern Toad and Green Treefrog. Based on observations by scientists and members of the general public, adult Cuban Treefrogs appear to be impacting some species of Florida's native wildlife through competition for food and shelter. As scientists continue to conduct 
research on the ecological impacts of Cuban Treefrogs we will develop a better understanding of the effects that this invasive frog is having on Florida's environment.

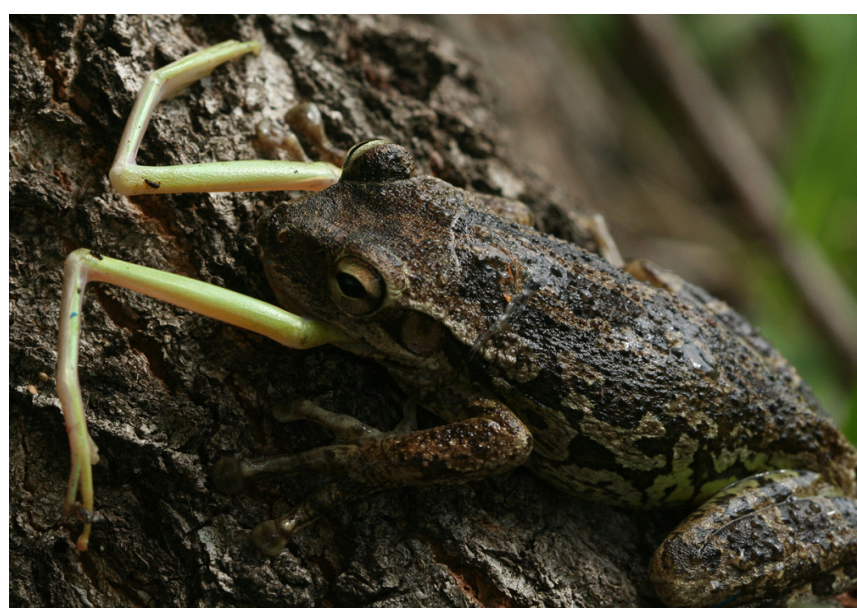

Figure 4. Cuban Treefrogs eat Florida's native treefrogs. (Note the legs of a native Green Treefrog protruding from the mouth of a predaceous Cuban Treefrog). Credits: Brent Anderson, 2006

\section{Quality of Life Impacts}

Cuban Treefrogs thrive in human-modified landscapes, such as urban and suburban communities. As a result, they are having impacts on the quality of life of Floridians. Cuban Treefrogs prefer tight, enclosed spaces in which they seek shelter during the day. Homes and buildings provide these shelters, as well as a food source. Because of the combination of abundant places to hide, consistent food sources, and adequate breeding sites provided by human dominated landscapes, Cuban Treefrog populations can become quite dense. This creates a situation in which these frogs can become a real nuisance for people.

Cuban Treefrogs are "sit-and-wait" predators. On warm nights in Florida where they occur it is common to encounter Cuban Treefrogs hanging on walls and windows near lighted areas as they sit and wait for insects to be attracted to the lights. As they feed, they defecate on the windows and walls and their fecal deposits can become unsightly over time, especially if there are a lot of frogs in the area. Furthermore, when a person enters or exits his or her home at night, Cuban Treefrogs that are waiting for an insect meal may be startled and as a result will occasionally jump onto people or into their homes through open doors. This can be a scary experience for a person who is afraid of frogs. Florida's native treefrogs rarely enter homes and buildings and do not cause the problems attributed to Cuban Treefrogs.

When they get into homes, Cuban Treefrogs can be especially annoying. Cuban Treefrogs can enter homes in a variety of ways. They may jump through open doors or windows, may inadvertently be brought into a house on an ornamental plant, or may enter via a home's plumbing system by gaining access through vent pipes on a roof. For information on how to exclude Cuban Treefrogs from homes and buildings see the forthcoming University of Florida/IFAS fact sheet on excluding frogs, snakes, and other wildlife from your home by Dr. Steve Johnson (http://edis.ifas.ufl.edu/TOPIC_A23708620). When Cuban Treefrogs gain access through vent pipes of a home plumbing system they usually end up in a bathroom. There are numerous instances where unsuspecting people have opened the lid to their toilet only to find a bug-eyed Cuban Treefrog staring back at them (Figure 5). Cuban Treefrogs have also been responsible for clogging sink drains.

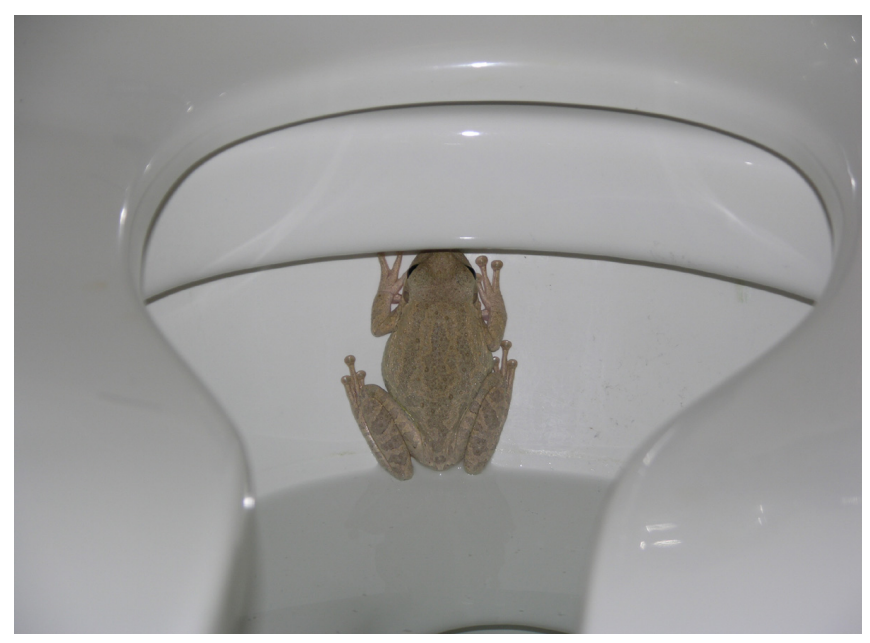

Figure 5. Cuban Treefrogs invade buildings and homes and sometimes find their way to toilets. Credits: Steve A. Johnson, University of Florida, 2006

Cuban Treefrogs breed primarily during Florida's warmest months, and breeding activity is influenced by rain. Like most frogs, male Cuban Treefrogs call to attract mates. When Cuban Treefrogs are concentrated in an area for breeding, their calls may be annoying to people that live near breeding sites, especially following heavy rains. 
Fortunately, the calls of individual frogs are not very loud. (See the list of resources at the end of this document for links to web sites where you can hear recordings of Cuban Treefrog calls). Because a single adult female may lay thousands of eggs, and many females will be present in any given pond, you may find numerous gelatinous masses of eggs floating on the surface of a decorative pond or swimming pool after a rainy night in the spring or summer. Left to develop into tadpoles, these egg masses may result in an onslaught of Cuban Treefrogs as they develop and metamorphose into small frogs in as little as 3-4 weeks (depending on the temperature of the water), further increasing the population of this invasive frog around people's homes.

Cuban Treefrogs may also be a nuisance to wildlife enthusiasts that set up nesting boxes to attract and benefit birds. Because Cuban Treefrogs prefer enclosed hiding spaces, they readily enter nest boxes erected for birds. Birds may be dissuaded from using nest boxes when they are invaded by Cuban Treefrogs. This appears to be the case for a homeowner near Sarasota, Florida, who erected boxes to attract Eastern Bluebirds and instead ended up providing hiding places for Cuban Treefrogs (Figure 6).

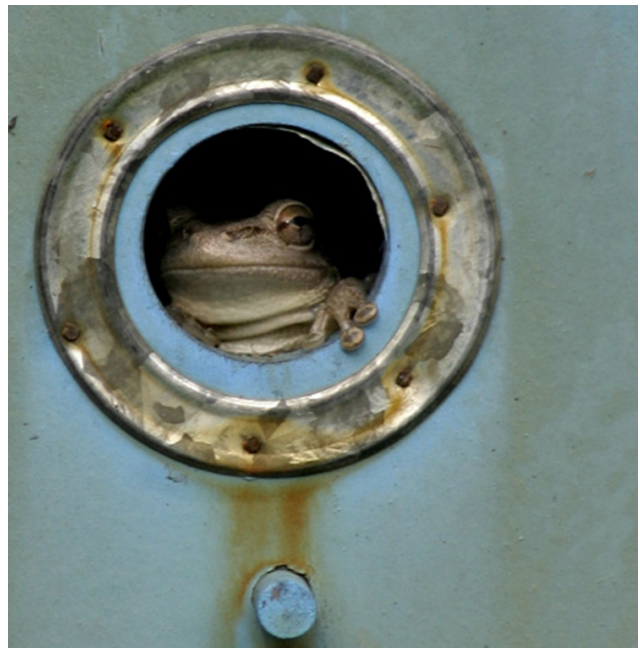

Figure 6. Cuban Treefrogs use birdhouses and nest boxes erected to benefit native wildlife. Credits: Barbara Zarella, 2005

Although they are not nearly as toxic as Cane Toads (also known as the invasive Bufo toad), Cuban Treefrogs have a sticky skin secretion that is extremely irritating to the mucous membranes of people, such as the eyes and nose. The secretions cause a burning and itching sensation that can last for more than an hour. This can be especially problematic for people who suffer from asthma or allergies, in which case full recovery from the ill effects of the frog's skin secretions may take several hours. Therefore, it is always a good idea to wash your hands after handling a Cuban Treefrog. Even though there do not appear to be any documented deaths or serious injuries of pets from ingesting or attempting to eat a Cuban Treefrog, dogs and cats should be kept away from these noxious frogs.

\section{Economic Impacts}

Unlike many invasive insect pests and invasive plants, Cuban Treefrogs do not currently appear to be having any large-scale negative affects on Florida's economy. Nonetheless, they are known to get into transformer boxes and electrical switches (Figure 7) and occasionally cause short-circuits. This increases maintenance costs for electrical utility companies, and power to some customers in central Florida has been interrupted as a result of short-circuits in "disconnect switches" caused by Cuban Treefrogs. As Cuban Treefrog populations continue to expand, this may eventually be a large-scale issue and research is currently underway at the University of Florida to test a potential chemical deterrent for Cuban Treefrogs.

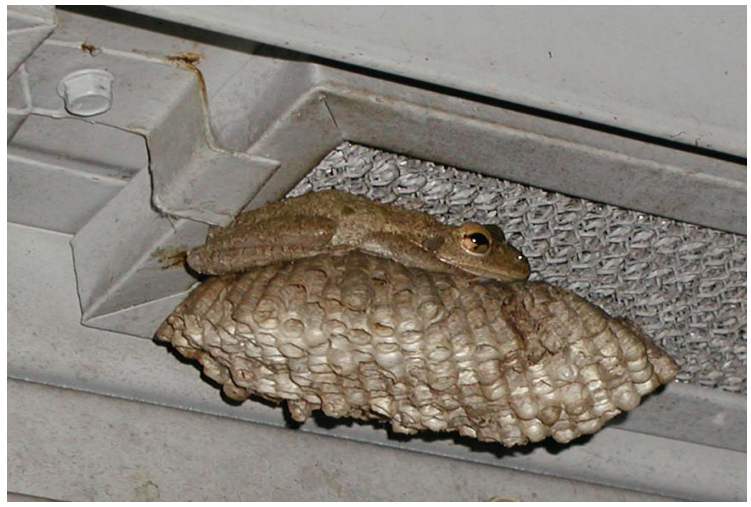

Figure 7. Cuban Treefrog perched on a wasp nest attached to a 12,470 volt substation circuit breaker. Fortunately, this frog did not cause a short-circuit. Credits: Steve Perkins, 2006 


\section{What You Can Do}

There are several things Floridians can do to help manage invasive Cuban Treefrogs and to help scientists at the University of Florida track their spread in the state and elsewhere. You can help advance our knowledge about the geographic distribution of this invasive species by reporting your sightings (see below) of Cuban Treefrogs. As Cuban Treefrogs continue to expand their range, precise tracking of their status and distribution is imperative for decision-making and resource management. In addition to helping track the expansion of their range, you can also help manage this invasive species in and around your yard. Because Cuban Treefrogs eat our native frogs and other wildlife, it is important that we take action to manage them and reduce their negative impacts on our native ecology. Managing Cuban Treefrogs will also help reduce their negative impacts on the quality of life of Floridians. Benefits will be greatest in the immediate area around where the frogs are managed (i.e., your yard). See below for suggestions on how to reduce the negative effects caused by Cuban Treefrogs on Florida's native wildlife and your quality of life.

\section{Report the Presence of Cuban Treefrogs}

To report the presence of Cuban Treefrogs on your property, please send an e-mail message to Dr. Steve A. Johnson of the University of Florida at tadpole@ufl.edu. Please be sure to include the county where the frog was seen and a street address of the location (for mapping purposes). When possible, please take a digital picture of the frog and include the image as an attachment in your e-mail message. This will allow Dr. Johnson to positively identify the frog and provide a confirmed record for our archives.

\section{Manage Cuban Treefrogs Around Your Home}

Because of the destructive effects of invasive Cuban Treefrogs on Florida's native species, as well as the problems they cause for people, we recommend that Cuban Treefrogs be captured and humanely euthanized. Before you euthanize a Cuban
Treefrog be sure that you are positive about its identification (please refer to the forthcoming University of Florida/IFAS fact sheet on Florida's treefrogs by Dr. Steve Johnson at http://edis.ifas.ufl.edu/TOPIC_A23708620).

To humanely euthanize a Cuban Treefrog you must first capture it; there are several effective methods for doing this. The first is to simply grab the frog from a window, wall, or other perch site. Be sure to wear rubber gloves or use a plastic grocery bag as a glove. Quickly and decisively approach the frog and with a continuous, swift movement firmly grab the frog (Figure 8).

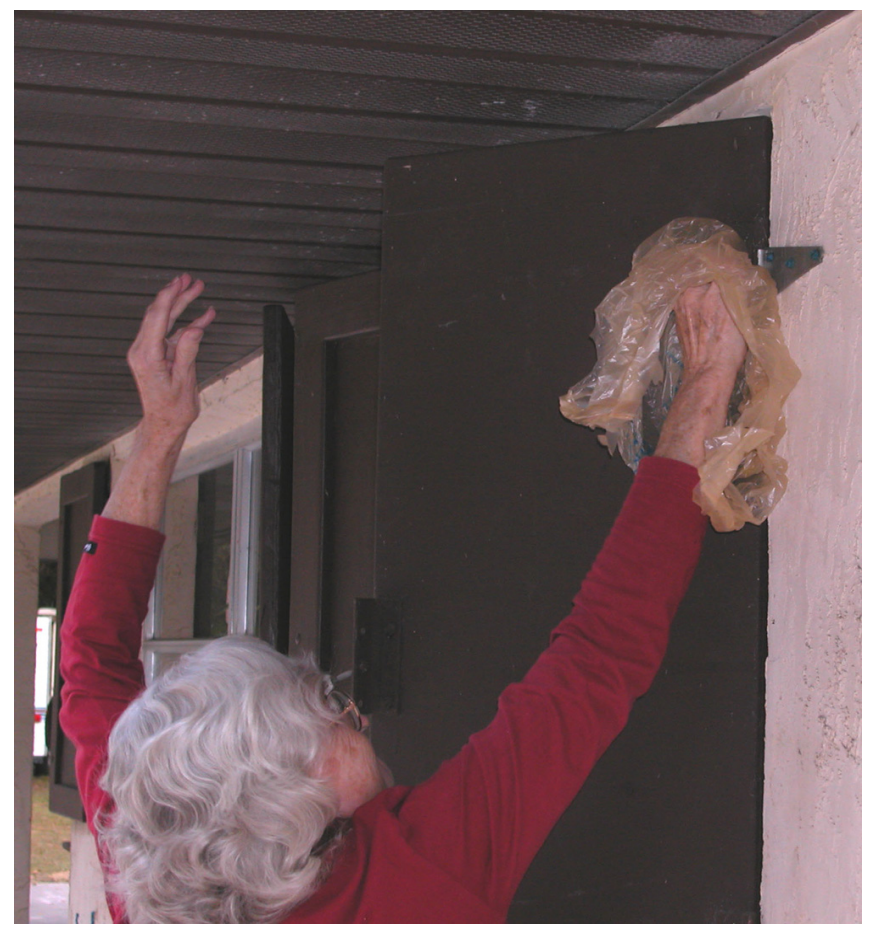

Figure 8. A central Florida homeowner helps manage Cuban Treefrogs by capturing and euthanizing this invasive species. Credits: Steve A. Johnson, University of Florida, 2006

Another way to capture Cuban Treefrogs in order to eliminate them from your property is to attract the frogs to hiding places where they can be easily captured and removed. To do this, simply place short sections of PVC pipe in the ground around your home and garden (Figure 9; cut 1 1/2 in. diameter PVC into approx $3 \mathrm{ft}$. long sections). Cuban Treefrogs, and hopefully native treefrogs too, will show up in the pipes in as little as a few days, depending on the weather and the density of frogs in your immediate area. In some situations it may take 
several weeks for frogs to find the pipes. To remove a frog from a pipe, simply pull the pipe from the ground and place a clear sandwich-bag over one end. Insert a broom handle or other "plunger" device in the other end and scare the frog into the sandwich bag. Once in the bag the frog can be examined closer to ensure that is an invasive Cuban Treefrog, and not a native species. Euthanize Cuban Treefrogs as described below and release native frogs back into the pipe. PCV pipes also provide great artificial habitats for native treefrogs and can help enhance the wildlife value of your garden. For more details on using PVC pipes to provide habitat for native treefrogs see the forthcoming University of Florida/IFAS fact sheet on attracting treefrogs to your Florida friendly yard by Dr. Steve Johnson (http://edis.ifas.ufl.edu/TOPIC_A23708620).

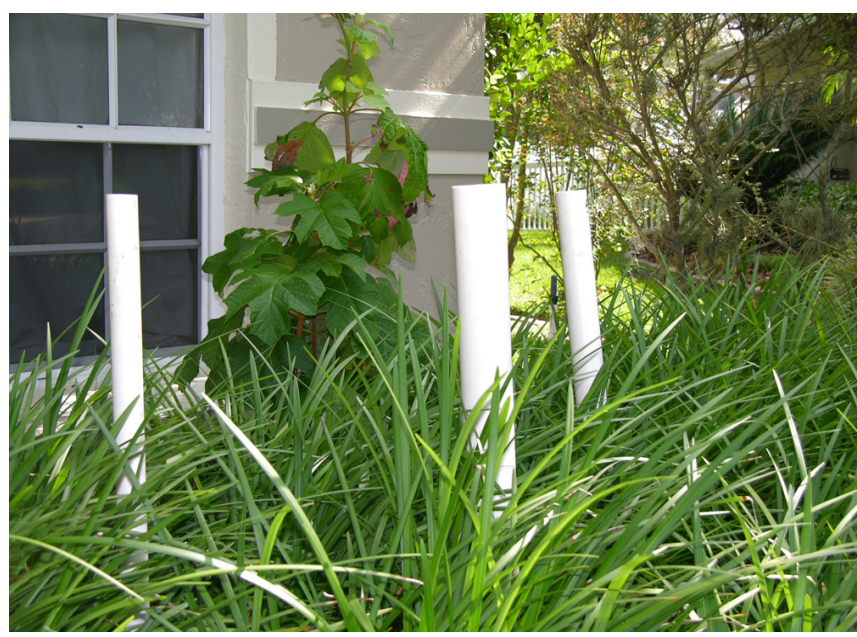

Figure 9. PVC pipes installed in your yard will attract native treefrogs and Cuban Treefrogs. Credits: Steve A. Johnson, University of Florida, 2007

To euthanize a Cuban Treefrog, hold the frog firmly in your hand and apply a 1 inch bead of benzocaine ointment along the back of the frog. Benzocaine ointment is a topical anesthetic (a numbing agent) used to treat skin pain (e.g., from sunburn) and itching as well as toothaches and sore throats. There are a variety of name brand and generic versions that are available over-the-counter in a tube or spray. If you are able to, using a gloved finger spread the ointment out on the frog's back. Alternatively, you could use a benzocaine spray. Once the ointment or spray is applied, place the frog in a plastic grocery bag or a sealable sandwich bag for 15-20 minutes so that the benzocaine has a chance to render the frog unconscious (be sure to seal the bag or tie it closed). After the bezocaine has anesthetized the frog, place the bag in a freezer overnight to ensure that the frog is dead and then throw it out in your trash. If you are unable to apply benzocaine to the frog, you can simply put it in a plastic bag, seal or tie the bag shut, then place it in the freezer overnight - dispose of the bag and the frog in the trash the next day. Do not throw a bagged frog into the trash without euthanizing it first. Remember, Cuban Treefrogs have a noxious skin secretion so be sure to wash your hands thoroughly after you handle the frog, even if you wear gloves or use a plastic bag.

You can also reduce the negative impacts of Cuban Treefrogs by eliminating eggs and potential breeding sites. Monitor ornamental ponds for Cuban Treefrog egg masses during the spring and summer, especially after heavy rains. Be sure to check for eggs the morning following rain so the eggs do not have time to hatch. Use a small-mesh aquarium net to scoop out masses of Cuban Treefrog eggs floating on the surface of the pond and simply discard them on the ground to dry out. Properly maintain swimming pools so they are not attractive to Cuban Treefrogs, and dump out stagnant water that accumulates in various objects around your yard (e.g., ice coolers, buckets, etc.). This will also help eliminate breeding sites for mosquitoes.

\section{Additional Sources of Information}

There are a variety of books, guides, and web sites that we recommend for additional information on treefrogs and other amphibians in Florida. These books are available at larger bookstores and online.

Carmichael, P., and W. Williams. 2004. Florida's Fabulous Reptiles and Amphibians. Tampa, FL: World Publications. 120 pp. ISBN: 0-911977-11-2. This is an excellent "coffee table" book with wonderful pictures and good information.

Conant, R., and J.T. Collins. 1998. Reptiles and Amphibians: Eastern/Central North America. Boston, MA: Houghton Mifflin Company. 450 pp. ISBN: 0-395-90452-8. This is the standard field guide for many herpetologists and covers hundreds of species of amphibians and reptiles. 
Meshaka, W. E., Jr., B.P. Butterfield, and J.B.

Hauge. 2004. The Exotic Amphibians and Reptiles

of Florida. Malabar, FL: Krieger Publishing

Company. 155 pp. ISBN: 1-57524-042-4. This book

contains species accounts for most of the

nonindigenous species of amphibians and reptiles in

Florida.

Florida Wildlife Extension website

http://www.wec.ufl.edu/extension/ -- Click the link to "Wildlife Information" then find the link to the

"Frogs \& Toads of Florida" page. This website contains images of all of Floridas frogs as well as recordings of the calls of most species, including the Cuban Treefrog. There are also links to many other useful wildlife related resources at the site.

\section{Dr. Steve Johnson's website}

http://ufwildlife.ifas.ufl.edu/ --Click the Extension link then follow the link for "Frogs and Toads".

Here you will find many digital images of Cuban Treefrogs and native treefrogs to aid with identification of these species. 